\title{
Three-dimensional Finite Element Analysis of Stress Distribution in Composite Resin Cores with Fiber Posts of Varying Diameters
}

\author{
Kazuhiko OKAMOTO, Teruo INO, Naoki IWASE, Eitaroh SHIMIZU, Megumi SUZUKI, Goh SATOH, \\ Shuji OHKAWA and Masanori FUJISAWA \\ Division of Prosthodontics, Department of Restorative and Biomaterials Sciences, Meikai University School of Dentistry, \\ 1-1 Keyakidai, Sakado, Saitama 350-0283, Japan \\ Corresponding author, Kazuhiko OKAMOTO; E-mail: okamoto@dent.meikai.ac.jp
}

Received June 28, 2007/Accepted August 6, 2007

\begin{abstract}
Using three-dimensional finite element analysis (3D-FEA), stress distributions in the remaining radicular tooth structure were investigated under the condition of varying diameters of fiber post for fiber post-reinforced composite resin cores (fiber post and core) in maxillary central incisors.

Four 3D-FEA models were constructed: (1) fiber post $(\phi 1.2, \phi 1.4$, and $\phi 1.6 \mathrm{~mm})$ and composite resin core; and (2) gold-cast post and core.

Maximum stresses in the tooth structure for fiber post and core were higher than that for gold-cast post and core. In the former models, stresses in the tooth structure as well as in the composite resin were slightly reduced with increase in fiber post diameter.

These results thus suggested that to reduce stress in the remaining radicular tooth with a large coronal defect, it is recommended to accompany a composite resin core with a fiber post of a large diameter.
\end{abstract}

Keywords: Finite element analysis, Composite resin core with fiber post, Fiber post diameter

\section{INTRODUCTION}

In the management of endodontically treated teeth, abutment construction is performed followed by prosthetic crown restoration. However, root fractures are occasionally observed in endodontically treated teeth. It has been reported that preserving the coronal tooth structure is effective - and essential - for preventing such fractures ${ }^{1-3)}$. However, an endodontically treated tooth usually has only a radicular tooth structure with a large coronal defect. In such cases, the use of a post is indispensable for the retention of the core. It has been advocated that the use of a metal post and core is effective in retaining an artificial crown for teeth with a large coronal defect ${ }^{4}$.

Recently, however, it was reported that metal post and core systems have an elastic modulus higher than that of root dentin. As a result, dentin stresses were concentrated around the post apex ${ }^{5}$. A root fracture, if it occurs, is usually vertical and unrepairable ${ }^{3,6,77}$. On the other hand, Newman et al. ${ }^{8)}$ found that a composite resin core with a fiber post prevented severe root fractures, because its elastic modulus is similar to that of root dentin. As reported by $\mathrm{Gu}$ et $a l .{ }^{9)}$ and Barjau-Escribano et al. ${ }^{10)}$, its root fracture patterns were different from that observed in the root with metal post and core. In particular, the failure mode allowed for further repair of - and hence reuse of - abutment teeth.

To date, many studies on fracture strength and fracture pattern have been conducted ${ }^{1,3,6-10)}$. Most of these studies focused on investigating stress distribution in the root dentin ${ }^{5,10-16)}$ of abutment teeth restored with a post and core, and also the effect of prefabricated post materials for reinforcement of the composite resin core ${ }^{17}$. Thus far, only a few studies focused on the influence of prefabricated post diameter on stress distribution in the remaining radicular tooth structure ${ }^{15)}$.

The purpose of this study, therefore, was to clarify - by means of three-dimensional finite element analysis (3D-FEA) - the effect of fiber post diameter on stress distribution in the remaining radicular tooth.

\section{MATERIALS AND METHODS}

Eight-node, isoparametric brick elements were constructed for 3D-FEA of a model simulating a maxillary central incisor restored with a post and core and a porcelain-fused-to-metal crown, including periodontal tissues. The unilateral side of mirror symmetry was analyzed (Fig. 1). The total number of nodes of the analysis model was 6,418, and the number of elements was 5,406. The mechanical properties of each material used for analysis are shown in Table 1 . The coronal structure was removed at the cervical line.

A static load $(100 \mathrm{~N})$ was applied to the model as a masticatory force from the palatal plane to the incisal edge at an angle of 45 degrees. The XYZ directions were restricted to the base and mesiodistal side, and the mesiodistal direction was restricted to the plane of mirror symmetry. For analysis, a finite element program for linear elastic materials ${ }^{18)}$ and a 


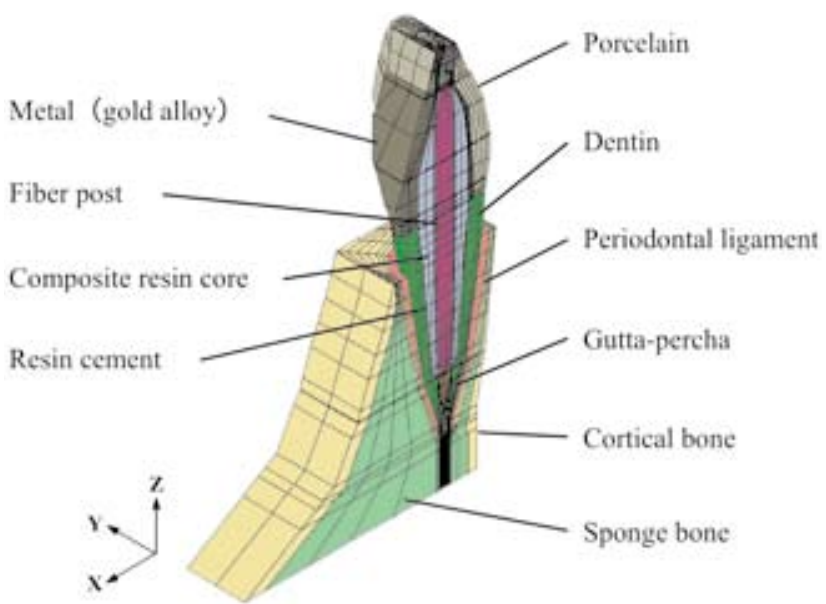

Fig. 1 FEA model of a maxillary central incisor restored with a post and core.

Table 1 Mechanical properties of materials in FEA model

\begin{tabular}{lcc}
\hline \multicolumn{1}{c}{ Material } & Young's modulus & Poisson's ratio \\
\hline Porcelain & 69,000 & 0.28 \\
Metal (gold alloy) & 95,000 & 0.33 \\
Dentin & 18,600 & 0.31 \\
Cortical bone & 13,700 & 0.30 \\
Sponge bone & 1,370 & 0.30 \\
Periodontal ligament & 6.9 & 0.45 \\
Gutta-percha & 0.69 & 0.45 \\
Fiber post & 45,000 & 0.25 \\
Composite resin & 16,600 & 0.24 \\
Resin cement & 95,000 & 0.30 \\
\hline
\end{tabular}
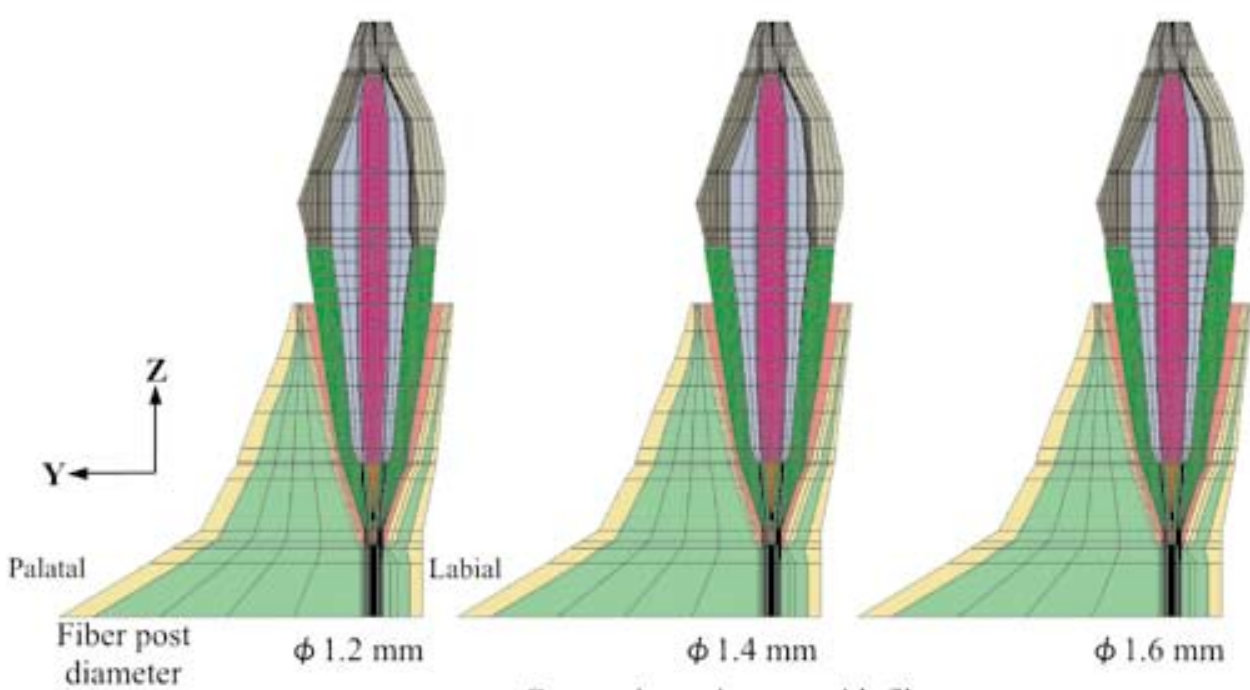

Composite resin core with fiber post workstation (Express5800, NEC Corp., Tokyo) were used.

Length of the post was $2 / 3$ that of root length, and the width was $1 / 2$ that of root width at the cervical line level. The model simulated two post and core systems: one model used a gold-cast post and core (i.e., metal post and core) and the other was a composite resin core with a fiber post (i.e., fiber post and core). To evaluate the latter, it entailed fiber posts of varying diameters $(\phi 1.2,1.4$, and 1.6 $\mathrm{mm}$ ) and a composite resin core, which bore a post shape of the same size (Fig. 2). All the materials were assumed to be homogeneous, isotropic, and linearly elastic.

At the initial stage, the bonding condition at the interface between the dentin and the post and core was assumed to be a complete bonding achieved with resin cement (100 $\mu \mathrm{m}$ in thickness). The principle stress that occurred in the elements simulating the luting cement layer was converted into force components parallel or vertical to the dentin interface. Bonding was assumed to have failed when any force component in the luting cement layer was higher than that of shear bond strength (25 MPa) or tensile bond strength (15 MPa), which was parallel and vertical to the interface respectively. The elastic modulus and Poisson's ratio were changed to $1.0 \mathrm{MPa}$ and 0.35. These calculations were repeated until none of the elements in the cement layer showed a value over the given criteria. Then, stresses occurring in the remaining radicular tooth structure and post region at convergence were calculated as von Mises stress equivalents.

Fig. 2 Models simulating the different post diameters and materials. 


\section{RESULTS}

Maximum stress in the remaining radicular tooth structure for fiber post and core models were observed on the inner side of the proximal wall at the level of the cervix irrespective of fiber post diameter (Fig. 3). As for the metal post and core, it was observed on the inner side around the post apex (Fig. 3). The fiber post and core models showed larger maximum von Mises stress values occurring in the remaining radicular tooth structure than that of the metal post and core, and the maximum stress was slightly reduced with an increase in fiber post diameter (Fig. 4).

For the fiber post and core models, stress concentration in the post region was observed at the interface between the lingual side of the fiber post and the core (Fig. 5). For the metal post and core, stress concentration was observed at the outer layer on the labial and lingual sides of the post from the
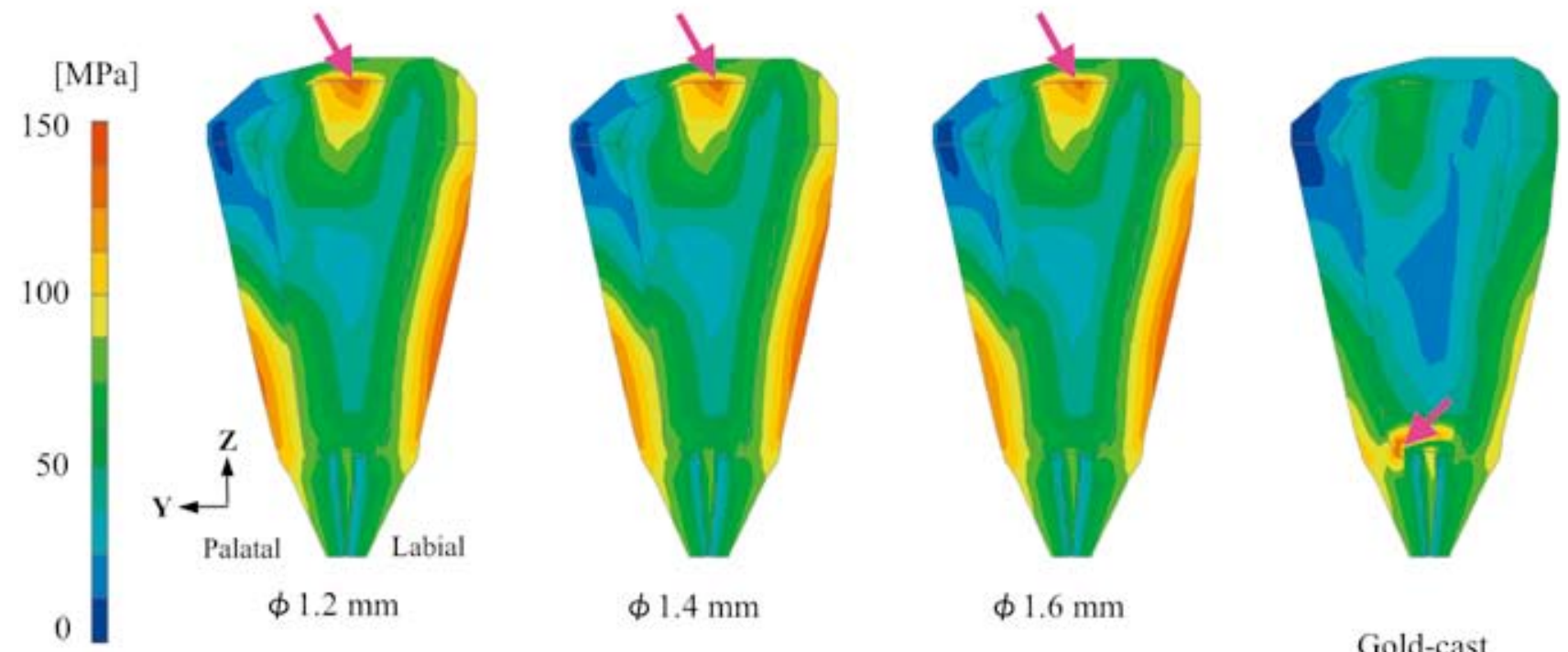

Composite resin core with fiber post

Gold-cast post and core

Fig. 3 Distribution of von Mises stresses in the remaining radicular tooth structure.

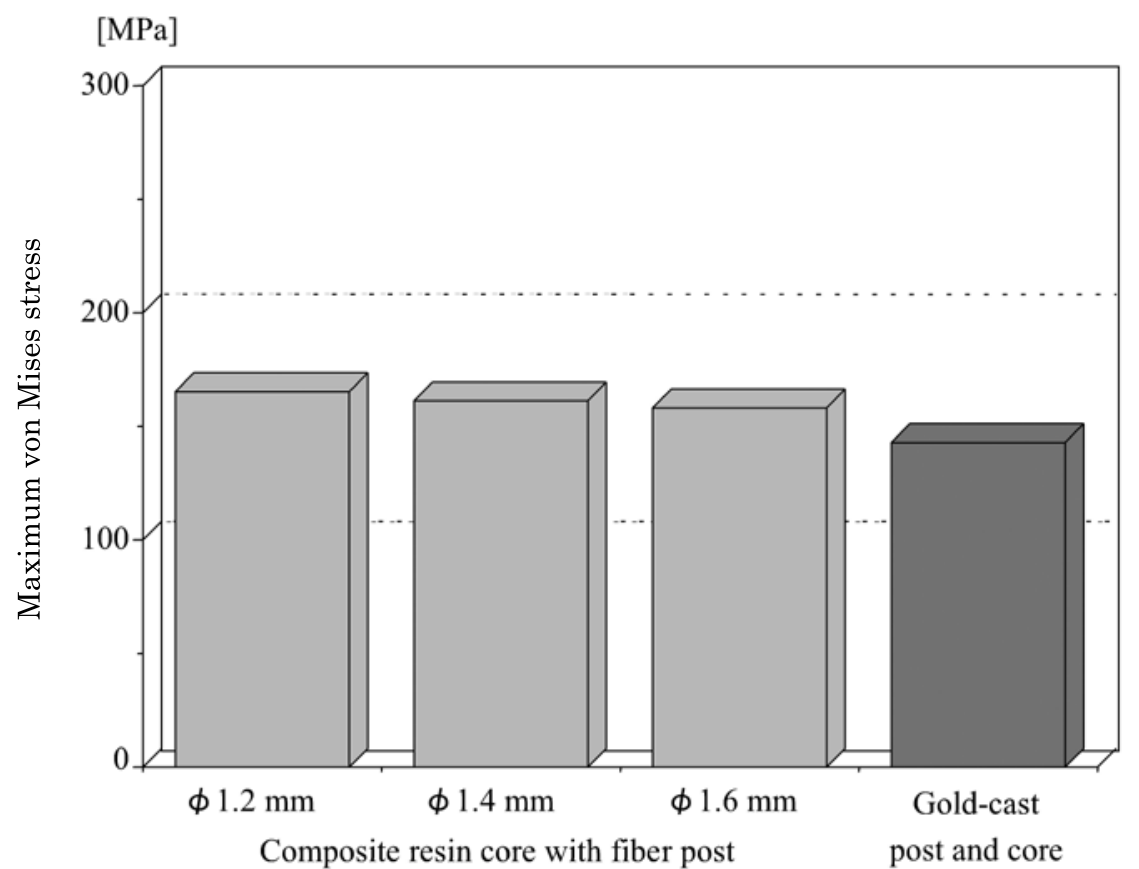

Fig. 4 Maximum von Mises stresses occurring in the remaining radicular tooth structure. 

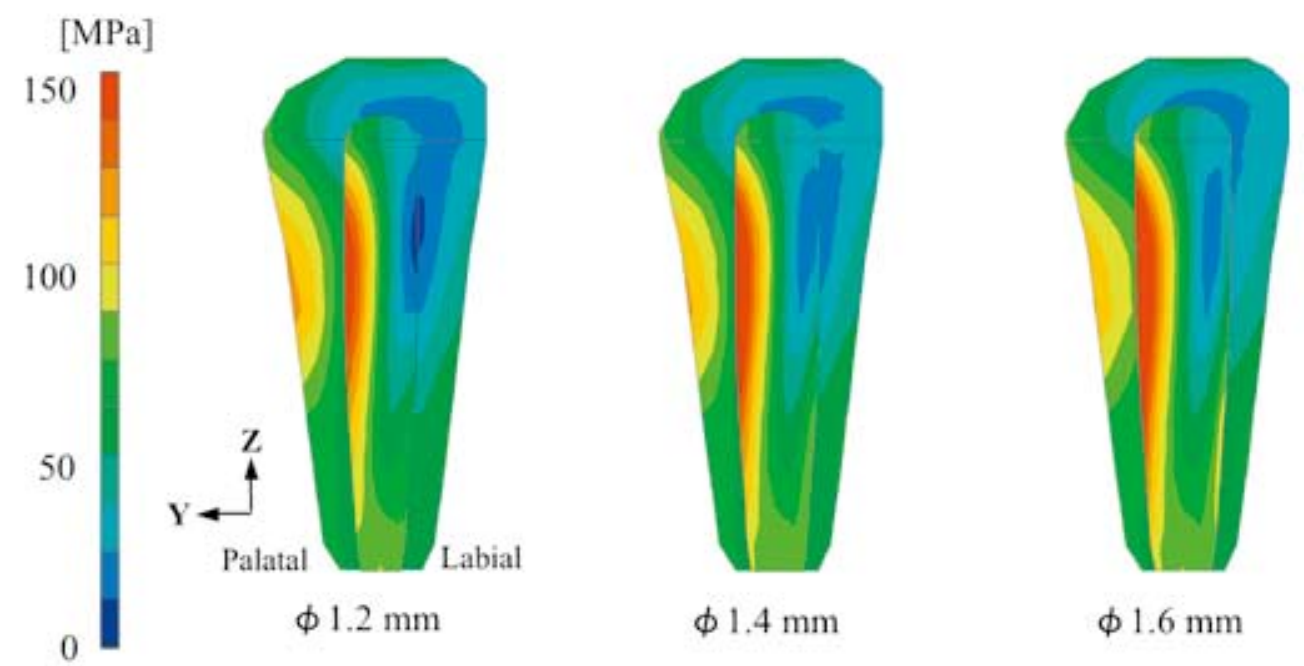

\section{Composite resin core with fiber post}

Gold-cast

post and core

Fig. 5 Distribution of von Mises stresses in the internal area of the post.

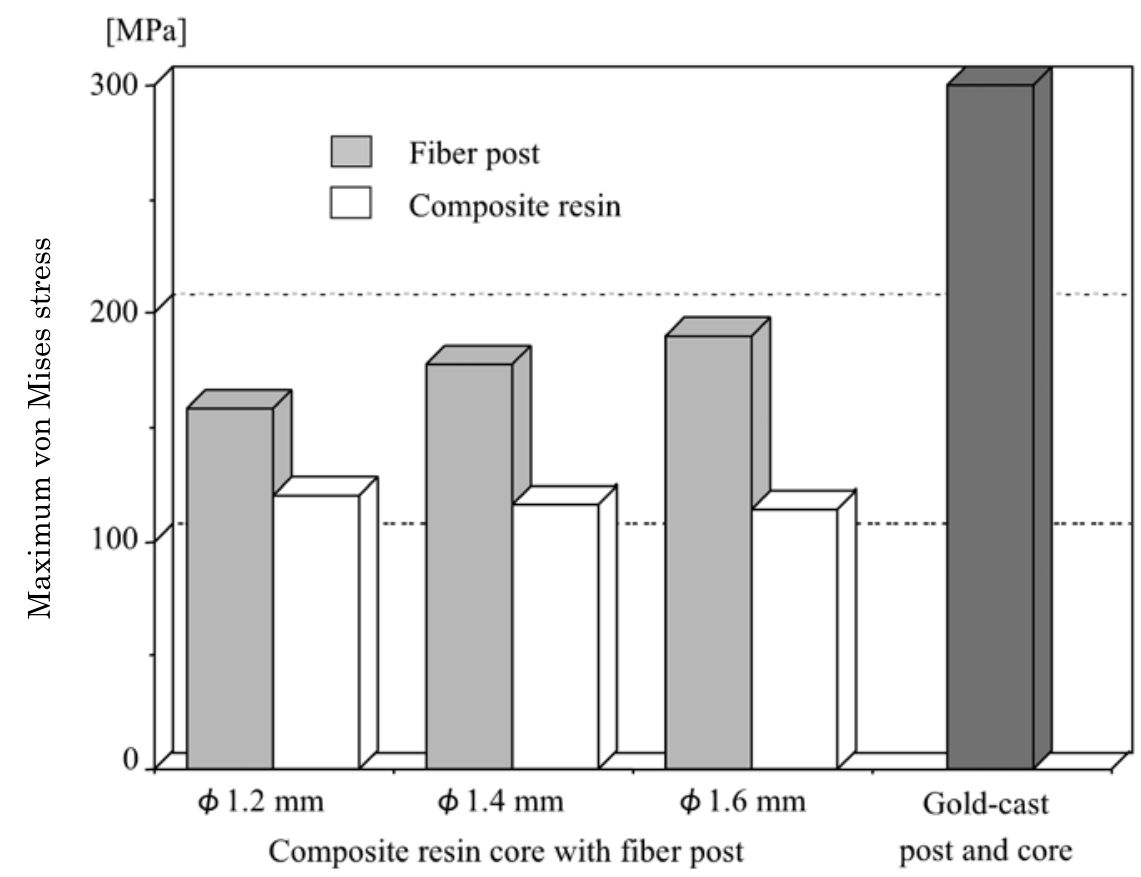

Fig. 6 Maximum von Mises stresses occurring in the internal area of the post.

cervix to the root apex area excluding the middle area of the post (Fig. 5). In the composite resin of the fiber post and core models, the maximum von Mises stress was slightly reduced with an increase in fiber post diameter. On the contrary, the maximum von Mises stress in the fiber post slightly increased with an increase in fiber post diameter (Fig. 6).

\section{DISCUSSION}

In clinical practice, when a metal post and core is inserted into an abutment tooth with a defect in its coronal tooth structure, root fracture often occurs ${ }^{19}$. The metal post and core has a high elastic modulus and fracture strength. When the root fracture occurs on the tooth restored with a metal post and core, tooth extraction is frequently the end result and only resolution ${ }^{3,6,7)}$. On the contrary, the fiber post shows mechanical properties and an elastic modulus similar to those of root dentin. These favorable features help to avoid catastrophic root fractures even when the abutment tooth is severely damaged ${ }^{8-10)}$. 
Although many types of fiber posts are currently available on the market, only a few studies have investigated on the effect of fiber post diameter on stress distribution in the root dentin. Therefore, this study was undertaken to evaluate the influence of fiber post diameter on stress distribution in the remaining radicular tooth structure by using 3D-FEA.

\section{Experimental method}

Barjau-Escribano et $a l .{ }^{10)}$ reported that experimental results obtained from the fracture strength test corroborated the estimations from the finite element model. We also previously reported that the fracture modes observed in fracture strength tests were consistent with the location of maximum stress observed by finite element analysis ${ }^{16)}$. Since these reports showed the possibility - and credibility - of 3D-FEA as a suitable method for simulating stresses occurring in teeth restored with a post and core, we used this method for analysis of the stress distribution in the abutment teeth.

In abutment construction, different types of posts are used for the reinforcement of the bending strength of the composite resin core. Post materials with mechanical properties similar to those of root dentin have been reported to be significantly effective against root fractures ${ }^{7,10)}$. However, apart from the mechanical properties of post materials, the post diameter itself may affect post displacement and stress distribution in the remaining radicular tooth structure. Therefore, in our bid to analyze the influence of fiber post diameter on stress distribution, we selected fiber posts with commercially available sizes of $\phi 1.2,1.4$, and $1.6 \mathrm{~mm}$. A metal post and core made from a conventional type IV gold alloy was also assessed as a control.

Maximum von Mises stress and stress distribution in the remaining radicular tooth structure

Pierrisnard et al. ${ }^{12)}$ and Asmussen et al. ${ }^{15)}$ evaluated the maximum von Mises stress occurring in the remaining radicular tooth restored with a post and core, as determined by use of 3D-FEA, and reported that an increase in the elastic modulus of the post materials reduced the maximum von Mises stress in the remaining radicular tooth structure. Tanino et $a l .{ }^{14)}$ evaluated the internal stress in root dentin around the post apex by using 3D-FEA on teeth with a glass fiber post, titanium alloy post, or a stainless steel post. They observed that the maximum principle stress in dentin was reduced using posts with an elastic modulus that approximated that of dentin. In the present study, the maximum von Mises stress of the metal post and core model with a high elastic modulus also showed a low value, whereas that of the fiber post and core models with a low elastic modulus showed high values. These findings clearly suggested that the elastic modulus influenced the maximum stress distribution in the remaining radicular tooth structure.

On the other hand, the maximum von Mises stress slightly decreased with an increase in fiber post diameter. Asmussen et $a l .{ }^{15)}$ analyzed the maximum von Mises stress occurring in the radicular dentin by changing the post diameter of titanium alloys and observed a decrease in the maximum von Mises stress in the root dentin with an increase in post diameter. Similar results were obtained in this study despite differences in the mechanical properties of the post materials. It is conceivable that with an increase in fiber post diameter which led to an increase in surface area of the post, stress dispersion might have thus aided in reducing stress development in the remaining radicular tooth structure.

In the remaining radicular tooth structure, the maximum von Mises stress of fiber post and core were observed on the inner side of the proximal wall at the level of the cervix, irrespective of fiber post diameter. However, that of the metal post and core was observed in the inner side around the post apex. Komada et al. ${ }^{6}$ measured the fracture strength of teeth restored with a post and core using extracted teeth embedded in acrylic resin. They reported that root fracture lines were observed below the level of acrylic resin in all teeth with a cast post and core, but above the level of acrylic resin in $75 \%$ of the teeth with a resin core and a glass fiber post.

Concerning stress distribution in the remaining radicular tooth structure, stress concentration tended to occur around the post apex in the case of the metal post and core. This was because the metal post with a high elastic modulus was hard to bend and might have acted as a lever support. With fiber post and core, the modulus was similar to that of root dentin. As a result, their flexural property led to stress concentration on the inner side of the proximal wall at the level of the cervix. Taken together, these results showed that the elastic modulus seemed to affect the location of maximum stress development and the mode of root fracture.

Maximum von Mises stress and stress distribution in the post region

Regarding the post region, the maximum von Mises stress in the fiber post and core models were lower than that in the metal post and core and increased with an increase in fiber post diameter. In contrast, inside the composite resin, the maximum von Mises stress slightly decreased with an increase in fiber post diameter. Further, with the fiber post and core, stress distribution in the composite resin decreased with an increase in fiber post diameter. These findings suggested that the load applied to the tooth restored with a fiber post and core was mainly received 
by the internal area of the fiber post. Moreover, stress burden in the fiber post increased, as compared with its decrease in the internal area of the composite resin, with an increase in fiber post diameter.

Concerning stress distribution in the post region as a whole, stress concentration was observed at the interface between the lingual side of the fiber post and composite resin. However, in the case of the metal post and core, stress concentration was on the outer layer of the whole post on the lingual and labial sides at the region of the cervix through the root apex area, excluding the middle area of the post.

Separately, in a report by Pegoretti et al. ${ }^{13)}$, stress concentration was observed at the postdentin interface for the model restored with a goldcast post and core and in the cervix area for the model restored with a fiber post and core. It should be noted that abutment teeth are composed of a mixture of materials with an assortment of their own critical structures. When high stresses mentioned above occur in the weak materials, such as luting cements or their adhesive interface, the luting cement layer as an adjacent component material may fail. Although an increase in fiber post diameter increased the area of stress distribution, stress concentration was limited to the interface between the fiber post and the composite resin. Thus, the location of the maximum stress development for the fiber post and core differed from that of the metal post and core, which showed the maximum stress on the outer layer of the whole post. At this juncture, it should be highlighted that for the fiber post and core, local stress concentration in the whole post might not occur. This is purportedly so by virtue of the flexural property being a typical characteristic of fibers. Consequently, stress distribution might instead be determined by the internal area of the whole post.

Results of this study suggested that the use of a large-diameter fiber post $(\phi 1.6 \mathrm{~mm})$ and composite resin core was better than with a small diameter $(\phi 1.2,1.4 \mathrm{~mm})$ or gold-cast post and core. This recommendation was made on the ground of reducing stresses in the remaining radicular tooth with a large coronal defect.

\section{CONCLUSIONS}

By means of 3D-FEA, this study evaluated the influence of fiber post diameter on stress distribution in the remaining radicular tooth structure of a maxillary central incisor with a large coronal defect. The following results were obtained:

(1) The maximum von Mises stress in the remaining radicular tooth structure for the composite resin core with a fiber post was higher than that for gold-cast post and core. However, it became slightly reduced with increase in fiber post diameter.

(2) The location of maximum stress development in the remaining radicular tooth structure was on the inner side of the proximal wall at the level of cervix for the composite resin core with a fiber post. For the gold-cast post and core, it was on the inner side around the post apex.

\section{REFERENCES}

1) Akkayan B. An in vitro study evaluating the effect of ferrule length on fracture resistance of endodontically treated teeth restored with fiber-reinforced and zirconia dowel systems. J Prosthet Dent 2004; 92: 155-162.

2) Creugers NH, Mentink AG, Fokkinga WA, Kreulen CM. 5-year follow-up of a prospective clinical study on various types of core restorations. Int J Prosthodont 2005;18: 34-39.

3) Hu S, Osada T, Shimizu T, Warita K, Kawawa T. Resistance to cyclic fatigue and fracture of structurally compromised root restored with different post and core restorations. Dent Mater J 2005; 24: 225231.

4) Morgano SM. Restoration of pulpless teeth: application of traditional principles in present and future contexts. J Prosthet Dent 1996; 75: 375-380.

5) Tanino Y, Ijima Y, Kuroe T, Satoh N, Tachibana K, Mitamura Y, Ohata N. Stress analysis of restorations of endodontically treated teeth using the finite element method - Effects of the elastic modulus of fiber and metal posts. Hokkaido J Dent Sci 2004; 25: $10-18$

6) Komada W, Miura H, Okada D, Yoshida K. Study on the fracture strength of root reconstructed with post and core: alveolar bone resorbed case. Dent Mater J 2006; 25: 177-182.

7) Akkayan B, Gulmez T. Resistance to fracture of endodontically treated teeth restored with different post systems. J Prosthet Dent 2002; 87: 431-437.

8) Newman MP, Yaman P, Dennison J, Rafter M, Billy E. Fracture resistance of endodontically treated teeth restored with composite posts. J Prosthet Dent 2003; 89: 360-367.

9) $\mathrm{Gu} \mathrm{XH}$, Kern M. Fracture resistance of crowned incisors with different post systems and luting agents. J Oral Rehabil 2006; 33: 918-923.

10) Barjau-Escribano A,Sancho-Bru JL, Forner-Navarro L, Rodriguez-Cervantes PJ, Perez-Gonzalez A, Sanchez-Marin FT. Influence of prefabricated post material on restored teeth: fracture strength and stress distribution. Oper Dent 2006; 31: 47-54.

11) Ho MH, Lee SY, Chen HH, Lee MC. Three-dimensional finite element analysis of the effects of posts on stress distribution in dentin. J Prosthet Dent 1994; 72: 367-372.

12) Pierrisnard L, Bohin F, Renault P, Barquins M. Corono-radicular reconstruction of pulpless teeth: a mechanical study using finite element analysis. J Prosthet Dent 2002; 88: 442-448.

13) Pegoretti A, Fambri L, Zappini G, Bianchetti M. 
Finite element analysis of a glass fibre reinforced composite endodontic post. Biomaterials 2002; 23: 2667-2682.

14) Tanino $Y$, Higa M, Nishimura I, Ishida T, Ohata $\mathrm{N}$, Mitamura Y. Effects of forms and materials of endodontic post on stress distribution - A stress analysis using three-dimensional finite element method. J Biomechanics 2004; 25: 223-228.

15) Asmussen E, Peutzfeldt A, Sahafi A. Finite element analysis of stresses in endodontically treated, dowelrestored teeth. J Prosthet Dent 2005; 94: 321-329.

16) Okamoto K, Ino T, Iwase N, Shimizu E, Suzuki M, Satoh G, Sone M, Ohkawa S. Stress analysis by 3dimensional finite element method for optimal construction of abutment teeth - Influence of thickness of remaining radicular tooth structure and abutment construction method on the stress distribution. J Meikai Dent Med 2007; 36: 30-36.

17) Grandini S, Goracci C, Monticelli F, Tay FR, Ferrari M. Fatigue resistance and structural characteristics of fiber posts: three-point bending test and SEM evaluation. Dent Mater 2005; 21: 75-82.

18) Zienkiewicz OC, Taylor RL. The finite element method, 5th, Vol 1, The Basis. ButterworthHeinemann, Oxford 2000; 127-139.

19) Axelsson P, Nystrom B, Lindhe J. The long-term effect of a plaque control program on tooth mortality, caries and periodontal disease in adults. Results after 30 years of maintenance. J Clin Periodontol 2004; 31: 749-757. 